\title{
Antidepressant Potential of Diphenyl Dimethyl Bicarboxylate (DDB) in a Modified Forced Swimming Test
}

\author{
Amal M. Mahfoz \\ Department of Pharmacology and Toxicology, Faculty of Pharmacy, Modern University for Technology and \\ Information, 11585, Cairo, Egypt
}

\begin{abstract}
Biphenyl Dimethyl Dicarboxylate (DDB); is an analogue of schisandrin C, a component isolated from Fructus Schisandrae Chinensis. It is now attracting growing attention for its polypharmacotherapeutic properties as an antiviral, hepatoprotective, and immunomodulator. It's been additionally possessed neurobehavioral impacts on experimental animals. Thus, the wide use of DDB, along with its neurobehavioral impacts; has encouraged us to study its antidepressant potential using the forced swimming test (FST). Furthermore, the protocol for FST was modified by combining it with the tail suspension test. The effect of DDB (100 mg/kg/day, p.o., 7 days $)$ on combined technique was evaluated against fluoxetine $(15 \mathrm{mg} / \mathrm{kg}$, i.p., single dose). In the end, the brains were dissected; serotonin (5-HT), dopamine (DA), and norepinephrine (NE) levels were measured. The results demonstrated that DDB decreased immobility in FST time as fluoxetine. Furthermore, DDB elevated 5HT, NE, and DA. DDB may be useful as a supportive antidepressant drug in hepatic diseased patients. However, more clinical studies are needed to ensure its safety.
\end{abstract}

Keywords: Depression; fluoxetine; DDB; forced swimming test; tail suspension test.

*Correspondence | Amal M. Mahfoz ; Department of Pharmacology and Toxicology, Faculty of Pharmacy, Modern University for Technology and Information, 11585, Cairo, Egypt. Email: amal.mahfoz@mti.edu.eg

Citation | Mahfoz AM, 2021. Antidepressant potential of Diphenyl Dimethyl Bicarboxylate (DDB) in a modified forced swimming test. Arch

Pharm Sci ASU 5(1): 63-70

DOI: $10.21608 /$ aps.2021.76190.1060

Print ISSN: 2356-8380. Online ISSN: 2356-8399.

Received 23 May 2021. Accepted 31 May 2021.

Copyright: ${ }^{{ }^{2}} 2021$ Mahfoz. This is an open-access article licensed under a Creative Commons Attribution 4.0 International License (CC BY 4.0), which permits unrestricted use, distribution, and reproduction in any medium, provided the original author(s) and source are credited.

Published by: Ain Shams University, Faculty of Pharmacy

\section{INTRODUCTION}

Depression is the most widespread neuropsychiatric disease. It is characterized by a persistent low mood that passes a heavy burden to patients' families and society. While there are many effective antidepressant drugs, many of them cause patient intolerance and severe adverse effects as sexual dysfunction, cardiotoxicity, and sleep disorder. The effort is invested to develop a novel antidepressant with fewer side effects and better efficacy. Fluoxetine; a popular selective serotonin reuptake inhibitor (SSRI), the most prescribed class of antidepressants. Despite the extensive use of SSRIs; many depressed patients do not remit after initial mono-therapy [1-3] Traditional Chinese Medicines have been reported to be more safe and effective in many circumstances [4]. Dimethyl-4,4'-dimethoxy5,6,5',6'-dimethylene-dioxy-biphenyl-

2,2 'dicarboxylate, usually abbreviated as DDB is an analogue of schisandrin $\mathrm{C}$, a component isolated from Fructus Schisandrae Chinensis; a Chinese folk medicine traditionally used for its tonic, sedative, antitussive, antioxidants, and anticancer properties [5]. It is usually prescribed 
as hepatoprotective in patients suffering from either viral or drug-induced hepatitis [6,7]. Recently, DDB was shown to be a behaviorally active agent, it was reported to be linked to significant effects on the behaviour of depressed partners in the sensory contact model, plus an immunomodulatory potential [8].

It is hard to develop an experimental model that accurately mimics the symptoms of depression in patients. The forced swim test (FST) was first developed by Porsolt et al. (1977) [9] for the rat and mouse [10]. FST is the easiest, reproducible, and commonly used test for preclinical experimental assessment of antidepressants $[\mathbf{1 1}, \mathbf{1 2}]$. The original protocol of FST is dependent on the idea that rodents after the first escape movements have developed immobility if placed in a non-escapable cylinder of water (pretest). Then if they replaced it 24 hours later; they resumed this posture rapidly (test). The immobile position reflects the development of passive behavior (depression) that prevents the animal activity to handle stress [3]. Upon administration of an antidepressant agent in-between the 2 exposures, animals will swim (escape-directed behavior) for a longer time than after treatment by vehicle [13].

Tail suspension test (TST) is also a behavioral technique that allows fast evaluation of psychoactive drugs. It is a sensitive method, rapid (maximum $6 \mathrm{~min}$ ), and is painless for the animal. Mice were suspended by the end of the tail; the presence of immobility (absence of limb movements) was measured over a 6-min session [14].

The present work aimed to study the antidepressant potential of DDB against fluoxetine using the FST. Furthermore, the protocol for FST was modified by combining it with TST.

\section{MATERIALS AND METHODS}

\subsection{Drugs and dosage}

Fluoxetine $(15 \mathrm{mg} / \mathrm{kg})$ was obtained as a white powder from Sigma Company. It was prepared as a fresh solution in deionized water just before use. It was injected as a single i.p. dose, $1 \mathrm{~h}$ before behavioral tests.

DDB (100 mg/kg, p.o, 7 days) was obtained as a white crystalline powder from The Arab Company of Pharmaceutical and Medicinal Plants (MEPACO). It was suspended in distilled water using $1 \%$ Tween 80 as an emulsifying agent. Dosage preparation was performed just before administration.

The utilized dose level in the present work was in the range of the doses previously reported to result in behavioral changes or to produce immunomodulatory effects [8].

\subsection{Animals}

Adult male Swiss mice $(25 \pm 5 \mathrm{~g})$ were obtained from the animal house of the National Organization of Drug Control and Research (NODCAR). Animals were kept under standard laboratory animal housing conditions with free access to water \& a conventional diet. The protocol of the current study is in line with the international guidelines for use of laboratory animals; published by the US NIH. It is also approved by the research ethics committee for experimental and clinical studies at the Faculty of Pharmacy, Modern University for Technology and Information (permit number: ES 884).

\subsection{Experimental design}

Animals were divided into 5 groups, each group consists of 7 animals.

Group 1: Animals were subjected to TST.

Group 2: Animals were subjected to FST. 
Group 3: Animals were subjected to combination technique (TST+FST).

Group 4: Animals were treated with a single dose of fluoxetine $(15 \mathrm{mg} / \mathrm{kg}$, i.p.) and after $1 \mathrm{~h}$ they were subjected to the combination technique (TST+FST).

Group 5: Animals were treated with DDB (100 $\mathrm{mg} / \mathrm{kg}$, p.o.) daily for 7 consecutive days, and on the $8^{\text {th }}$ day they were subjected to the combination technique (TST+FST).

DDB administration took place at 2-3 p.m., The testing sessions took place at 10-12 a.m.

\subsection{Tests}

\subsubsection{The following behavioral tests were performed}

\subsubsection{The Tail Suspension Test (TST)}

Mice were suspended by the tail with adhesive tape (40 $\mathrm{cm}$ above the table) for 6 minutes. The session was videotaped, and the duration of immobility in seconds was recorded. Mice were considered immobile only when hung passively and completely motionless [15].

\subsubsection{The Forced Swimming Test (FST)}

Each mouse was placed separately in a cylinder $(12 \times 24 \mathrm{~cm})$ full with water to $12 \mathrm{~cm}$ height at $22-23{ }^{\circ} \mathrm{C}$. The session was recorded and the period of immobility; swimming; and straggling through the latest $3 \mathrm{~min}$ were assessed. Immobility was demonstrated by stopping straggling and moving only to continue floating on the water surface. After the $6 \mathrm{~min}$ of the test, the animal was removed and allows drying [16]. The FST technique is widely used to study depression behavior in mice, rats, dogs [17], and also in kids disconnected from their parents [18].

\subsubsection{Combination of TST\&FST}

For the sake of increasing the depressant potential of the FST, the effect of the combination of two techniques of depression (TST\&FST) was studied on the depressive state, and compared the influence of fluoxetine and DDB using this combination technique.

The animals were allowed to be suspended individually by their tail for 6 minutes (as the procedure mentioned above), then introduced to the FST apparatus for $6 \mathrm{~min}$. The FST session was recorded and the period of immobility; swimming; and straggling through the last $3 \mathrm{~min}$ of the FST were measured.

\subsubsection{Assay of brain neurotransmitters}

Brain catecholamine and serotonin were determined spectrophotoflurometrically according to Ciarlone (1978) [19] and Khalifa et al. (1997) [20] using Spectro-photoflourometer RF-5000 Shimadzu, Japan. Animals were decapitated with the least disturbance using large scissors and the brain was rapidly dissected on ice, weighed, and homogenized with acidified butanol in a glass homogenizer submerged in ice. The homogenate was then centrifuged at $2000 \times \mathrm{g}$ for $5 \mathrm{~min}, 2.5 \mathrm{~mL}$ of the supernatant was transferred to a test tube containing $1.6 \mathrm{ml}$ of 0.2 $\mathrm{N}$ acetic acid and $5 \mathrm{~mL}$ n-heptane. Tubes were then vortexed for $30 \mathrm{sec}$, centrifuged at $2000 \times \mathrm{g}$ for exactly $5 \mathrm{~min}$. The supernatant organic layer was discarded and $0.2 \mathrm{~mL}$ of the aqueous phase was transferred to a test tube to measure serotonin and another $1 \mathrm{mLwas}$ transferred to another test tube to measure NE and DA. To the test tube containing $0.2 \mathrm{~mL}$ aqueous phase, 1.2 $\mathrm{ml}$ of 4\% O-phthalaldehyde was added, mixed well then placed at boiling water bath for 10 minutes, cooled under tap water, and measured at $355 \mathrm{~nm}$ excitation and 470 emissions for the analysis of serotonin. To the $1 \mathrm{~mL}$ aqueous solution, $0.2 \mathrm{~mL}$ of $0.1 \mathrm{M}$ EDTA was added and mixed well. Catecholamines were oxidized using $0.1 \mathrm{~mL}$ iodine and oxidation reaction was terminated after exactly 2 min by adding $0.2 \mathrm{~mL}$ alkaline sulfite. The tubes were allowed to stand 
exactly 2 min then $0.2 \mathrm{~mL}$ of $5 \mathrm{~N}$ acetic acid was added and mixed. The mixture was placed in a boiling water bath for $2 \mathrm{~min}$, cooled, and measured at $380 \mathrm{~nm}$ excitation and 480 emissions for NE. Test tubes were replaced with the boiling water bath for 5 min more, cooled, and measured at 320 excitations and $375 \mathrm{~nm}$ emissions for determination of DA. The assay depends upon estimating the norepinephrine and dopamine flurophores formed after oxidation with iodine and measuring the serotonin flurophores formed by derivatization using O-phathalaldehyde at different excitation and emission wavelengths.

\subsection{Statistical analysis}

All groups were statistically compared using one-way ANOVA [21] followed by the Tuckey post hoc test. The Difference between means was considered significant when $\mathrm{P}<0.05$. Statistical analysis was performed using SPSS 16 analysis pack.

\section{RESULTS}

\subsection{Behavioral Analysis (Fig. 1): a. immobility, b. swimming, \& c. straggling time}

The results showed that the immobility time was significantly elevated in the group that passed by TST before FST (combination technique) more than the group passed FST directly (123.9\%). However, both swimming and struggling time significantly decreased in combined technique as compared to FST $(64.6 \%$, $58.2 \%)$. There was a similar effect of both Fluoxetine \& DDB on reducing the immobility time when compared to the combination technique.

\subsection{Neurotransmitters levels (Fig. 2)}

\subsubsection{Serotonin}

The results revealed that the whole brain's 5HT concentration was significantly decreased following application of either TST (83\%) or FST (67\%). Moreover, after the combination of the two techniques (TST+FST) the 5-HT level was significantly decreased less than the application of TST (55\%) or FST (67\%) alone. Treatment of the depressed mice with fluoxetine normalized 5-HT level of depressed mice. However, DDB treatment resulted in a significant elevation in 5-HT levels, as compared to the combined technique (184\%).
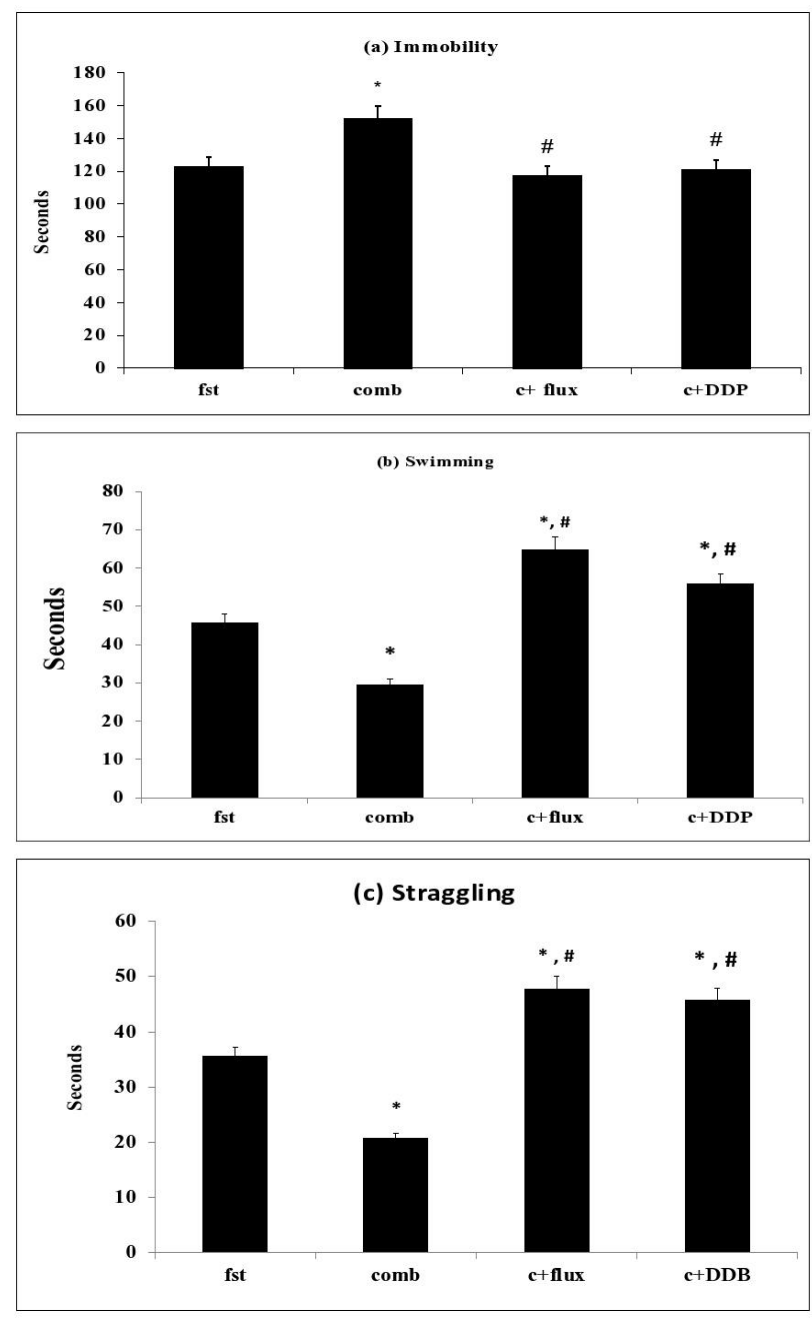

Fig. 1. Behavioral effects of the FST, combination technique, fluoxetine and DDB with combination technique $(n=6)$

a. Immobility time, b. swimming time, c. straggling time FST: forced swimming test, comb: combination technique, c+flux: combination technique plus fluoxetine and c+DDB: combination technique plus DDB

* Significant difference, at $\mathrm{P}<0.05$, from FST

\# Significant difference, at $\mathrm{P}<0.05$, from combination technique 


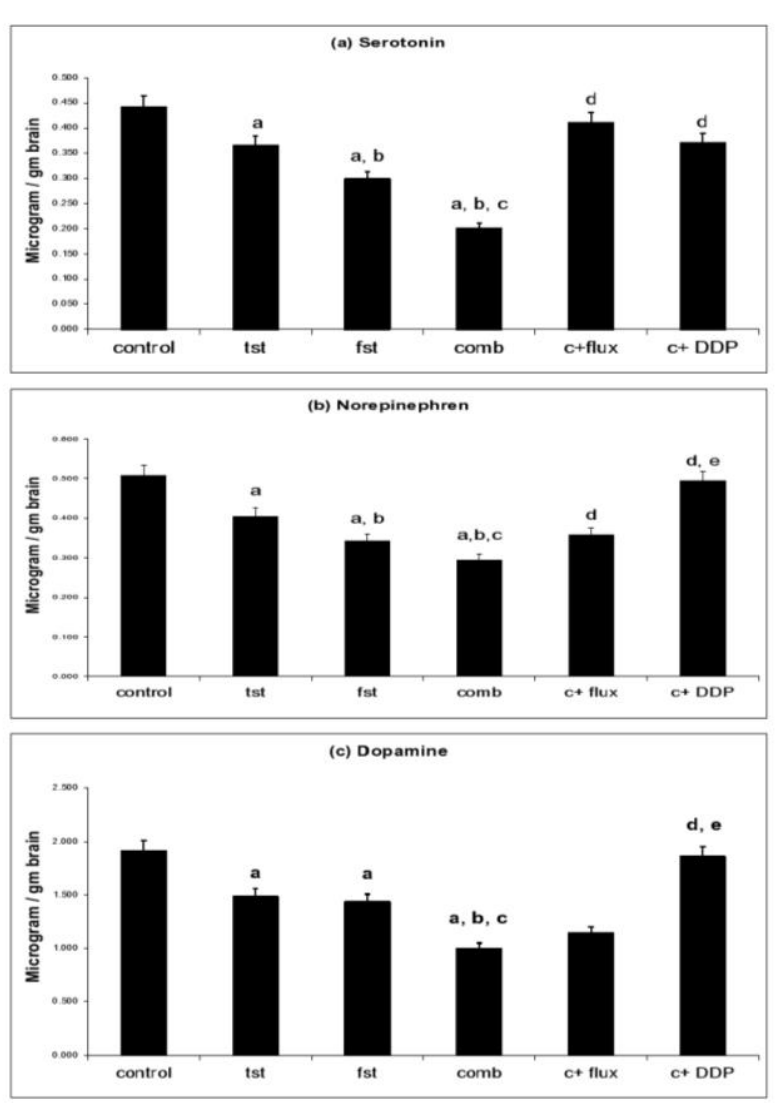

Fig. 2. Effects of the combination technique on neurotransmitters levels in the brain (a. serotonin, b. norepinephren and c. dopamine) with comparison to control and effects of fluoxetine and DDB in the combination technique on these neurotransmitters with comparison to the combined technique without treatments $(n=6)$

tst: tail suspension test, fst: forced swimming test, comb: combination technique, c+flux: combination technique plus fluxotine and c+DDB: combination technique plus DDB a Significant difference, at $\mathrm{P}<0.05$, from normal control $\mathrm{b}$ Significant difference, at $\mathrm{P}<0.05$, from tst. c Significant difference, at $\mathrm{P}<0.05$, from fst. d Significant difference, at $\mathrm{P}<0.05$, from comb e Significant difference, at $\mathrm{P}<0.05$, from $\mathrm{c}+\mathrm{fst}$

\subsection{Norepinephrine}

The combined technique produced a significant lowering in brain NE when compared to TST $(72.3 \%)$ or FST $(85.7 \%)$ alone. Fluoxetine resulted in a significant elevation in NE level, compared to the combined technique (121.8\%). In contrast, administration of DDB to animals subjected to combination technique
(TST+FST) nearly normalized brain NE level.

\subsection{Dopamine}

The obtained results declared that the DA level was significantly lowered in combination technique when compared to TST $(67.3 \%)$ or FST $(69.7 \%)$ alone. Administration of fluoxetine to animals subjected to the combination technique caused a non-significant increase in DA level. However, DDB treatment for 7 constitutive days induced a significant increase in DA level of mice exposed to combination technique (by $185.8 \%$ ) nearly to the normal level.

\section{DISCUSSION}

The present study aimed to determine if the combination of two models of depression, TST and FST affects the immobility time (depressive state). In addition to, comparing the antidepressant potential of a standard antidepressant drug; fluoxetine with DDB. As shown previously the immobility time in both FST and TST is a reflection of the depressed state in mice $[\mathbf{1 4}, \mathbf{2 2}]$. Concerning the combination of TST \& FST, both are well-known models of depression; the present results found that there was an increase in the immobility time in the FST in animals passing TST before FST than animal passing FST directly. The increase in immobility time reflects the increase in the depressed state [22]. This increase was accompanied by a significant decrease in swimming, straggling time, and whole-brain neurotransmitters: 5HT, $\mathrm{NE}$, and DA. These findings are similar to those obtained by several investigators $[23,24]$ who noticed that dysfunction in the 5HT concentration may causally related to major depressive disorder. The modified FST protocol in the current study allows so far the investigation of acute or chronic treatments and with lower doses of antidepressants or a lower number of animals. It may evaluate different types of antidepressant drugs acting by different mechanisms of action. 
The present results showed that the increase in immobility time after subjected the animals to the combination of the two behavioral tests (TST, FST) is decreased by acute treatment with fluoxetine; however, swimming time increased. This finding is consistent with previous findings $[25,26]$. This effect was accompanied by a significant increase in 5HT level in the whole brain of mice subjected to the combination technique and treated by fluoxetine; this finding is consistent with previous study which noticed that fluoxetine increased 5-HT in depressed animals. However, the results showed that injection of fluoxetine had little effect on NE or DA levels of depressed animals (mice subjected to the combination technique) [3].

Administration of DDB resulted in decreasing immobility time to a similar value as fluoxetine. This effect was accompanied by a significant increase in swimming, straggling time, and brain neurotransmitters. Dopamine is the major biogenic amine involved in the control of locomotor activity [27]. The clear effect of DDB on immobility time in the FST is correlated with a correspondent tendency to increase DA significantly in depressed mice. The utilized dose level of DDB in the present work $(100 \mathrm{mg} / \mathrm{kg}$ given orally) was in the range of the doses previously reported to result in behavioral changes or to produce immunomodulatory and hepatoprotective effects [6]. The neurobehavioral impacts of DDB were previously reported in enhancing the aggressiveness of the winner partner in the sensory contact model and to increase motor activity in the open field test. Furthermore, it decreased anxiety and increased exploratory tendency and curiosity [8]. It is reported that the monoaminergic hypothesis is the most accepted mechanism of depression by major depletion of brain 5HT, NE, and DA [4]. Furthermore, the antioxidant and antiinflammatory impact of DDB which is extracted from Schisandra Chinensis protects against central neurodegeneration \& neuroinflammation which have been reported in various CNS disorders [28-32]. So the antidepressant-like effect of DDB which was observed in the current study may be considered as a parallel to the observed changes in neurotransmitters and the increase in motor activity or to the decrease of anxiety as observed in the current study.

\section{Conclusion}

It could be concluded that the combination of TST and FST increases the sensitivity of FST for testing different antidepressant drugs. DDB is not an inert substance on the brain; it is maybe an antidepressant or antidepressant plus other neurobehavioral effects. So this study points to the need for other detailed complementary preclinical and clinical investigations to define the neuropharmacological potential of DDB.

\section{Declarations}

\section{Ethics approval and consent to participate}

Not applicable

\section{Consent to publish}

Not applicable

\section{Availability of data and materials}

The data generated or analyzed during this study all are included in the main manuscript.

\section{Competing interests}

The author declares that no competing interests exist.

\section{Funding statement}

No funding source was received

\section{REFERENCES}

1. Rush A. J., M. H. Trivedi, S. R. Wisniewski, A. Nierenberg, J. W. Stewart, D. Warden, et al., ava Acute and longer-term outcomes in depressed outpatients requiring one or several treatment steps: a STAR*D report Am. J. Psychiatr., 2006, 
163, pp. 1905-1917

2. Hillhouse TM, Porter JH. A brief history of the development of antidepressant drugs: from monoamines to glutamate. Exp Clin Psychopharmacol. 2015;23(1):1-21. doi:10.1037/a0038550.

3. Shazia Dawood, Samina Bano, Saira Bugti and Jai Kershan. Acute Fluoxetine Treatment Produce Anxiolytic Effects without Modulating Behaviour Response on Exposure to Forced Swimming Test. Saudi J Med Pharm Sci. 2019; 5(9): 785-790.

4. Yan T, Xu M, Wu B, Liao Z, Liu Z, Zhao X, et al. The effect of Schisandra chinensis extracts on depression by noradrenergic, dopaminergic, GABAergic and glutamatergic systems in the forced swim test in mice. Food Funct. 2016, 15;7(6):2811-9. doi: 10.1039/c6fo00328a. Epub 2016 May 26. PMID: 27225351.

5. Liu, T.Y.; Hwuna, Y.S.; Chao, T.W. and Chi, C.W.. Mechanistic study of the inhibition of aflatoxin b1induced hepatotoxicity by dimethyl-4,4 dimethoxy-5,6,5 ,6 -dimethylenedioxybiphenyl-

2,2.Cancer Lett. 1995,2;89(2):201-5. doi: 10.1016/0304-3835(95)03688-s.

6. Abdel-Hameid NA. Protective role of dimethyl diphenyl bicarboxylate (DDB) against erythromycin induced hepatotoxicity in male rats., Toxicol In Vitro., 2006, 4: 618-25.

7. Park E.Y.; Ki S.H.; Ko M.S.; Kim C.W.; Lee M.H.; Lee Y.S. et al., Garlic oil and DDB, comprised in a pharmaceutical composition for the treatment of patients with viral hepatitis, prevents acute liver injuries potentiated by glutathione deficiency in rats. Chem Biol Interact., 2005, 155 (1-2): 82-96.

8. Mahfoz, A.M. Social behavior effects of diphenyl dimethyl bicarboxylate (DDB) in the sensory contact model. Naunyn-Schmiedeberg's Arch Pharmacol., 2019, 392, 313-326. https://doi.org/10.1007/s00210-018-1580-7

9. Porsolt, R.D.; Le Pichon, M. and Jalfre, M. Depression: A new animal model sensitive to antidepressant treatments. Nature, 1977, 266: 730732.
10. Porsolt, R.D. Animal models for depression: Utility for transgenic research. Rev. Neurosci., 2000, 11: 53-58

11. John P. Redrobe; Yvan Dumonta; Alain Fournier; Glen B. Baker and R'emi Quirion. Role of serotonin (5-HT) in the antidepressant-like properties of neuropeptide Y (NPY) in the mouse forced swim test. Peptides, 2005, 26: 1394-1400.

12. Borsini, F. and Meli, A. Is the forced swimming test a suitable model for revealing antidepressant activity?. Psychopharmacol. (berl.), 1988, 94: 147160.

13. Lucki, I. The forced swimming test as a model for core and component behavioral effects of antidepressant drugs. Behav. Pharmacol., 1997, 8: 523-532.

14. Steru L, Chermat R, Thierry B, Simon P. The tail suspension test: a new method for screening antidepressants in mice. Psychopharmacology (Berl), 1985, 85: 367-370.

15. Gribkoff VK, Kaczmarek LK. The need for new approaches in CNS drug discovery: Why drugs have failed, and what can be done to improve outcomes. Neuropharmacology. 2017 Jul 1;120:1119. doi: 10.1016/j.neuropharm.2016.03.021. Epub 2016 Mar 12. PMID: 26979921; PMCID: PMC5820030.

16. Yankelevitch-Yahav R, Franko M, Huly A, Doron R. The forced swim test as a model of depressivelike behavior. J Vis Exp. 2015 Mar 2;(97):52587. doi: 10.3791/52587. PMID: 25867960; PMCID: PMC4401172.

17. Lima IMM, Peckham AD, Johnson SL. Cognitive deficits in bipolar disorders: Implications for emotion. Clin Psychol Rev. 2018 Feb;59:126-136. doi: 10.1016/j.cpr.2017.11.006. Epub 2017 Nov 21. PMID: 29195773; PMCID: PMC6404979.

18. Stroebe M. The Study of Security and Separation: An Unexpected Forerunner of Attachment Theory? Omega (Westport). 2019 Oct 14:30222819880706. doi: 10.1177/0030222819880706. Epub ahead of print. PMID: 31610736.

19. Ciarlone, A.F. Further modification of a 
fluorometric method for analyzing brain amines. Microchemical J., 1978, 23: 9-12.

20. Ewa K „edzierska, Lila D ،abkowska , Paweł Obierzy 'nski , Magdalena Polakowska, Ewa Poleszak, Piotr Wla 'z , Katarzyna Szewczyk and Jolanta Kotli 'nska. Synergistic Action of Sodium Selenite with some Antidepressants and Diazepam in Mice. Pharmaceutics 2018, 10, 270; doi:10.3390/pharmaceutics 10040270

21. Armitage, P. and Berry, G. Comparison of several groups. In statistical methods in medical research. $2^{\text {nd }}$ edition, Blackwell scientific publications, Oxford, 1987, pp. 186-213.

22. Porsolt R.D.; Anton G. and Daniel M. Behavioral despair in rats: a new model sensitive to antidepressant treatments. Eur J Pharmacol., 1978, 17:379-91.

23. Booij, L.; Van det Does, A.J. and Riedel, W.J. Monoamine depletion in psychiatric and healthy populations. Mol. Psych., 2003, 8: 951-973.

24. Ruhe, H.G.; Mason, N.S. and Schene, A.H. Mood is indirectly related to serotonin, norepinephrine and dopamine levels in humans: a meta-analysis of monoamine depletion studies. Mol. Psych., 2007, 12: 331-359.

25. Kroeze, Y., Peeters, D., Boulle, F. et al. Long-term consequences of chronic fluoxetine exposure on the expression of myelination-related genes in the rat hippocampus. Transl Psychiatry 5, e642 (2015). https://doi.org/10.1038/tp.2015.145.

26. Poleszak, E., Szopa, A., Bogatko, K. et al. Antidepressant-Like Activity of Typical Antidepressant Drugs in the Forced Swim Test and Tail Suspension Test in Mice Is Augmented by DMPX, an Adenosine $\mathrm{A}_{2 \mathrm{~A}}$ Receptor Antagonist. Neurotox Res 35, 344-352 (2019). https://doi.org/10.1007/s12640-018-9959-2

27. Dhuriya YK, Srivastava P, Shukla RK, Gupta R, Singh D, Parmar D, Pant AB, Khanna VK. Prenatal exposure to lambda-cyhalothrin alters brain dopaminergic signaling in developing rats. Toxicology. 2017 Jul 1;386:49-59. doi: 10.1016/j.tox.2017.04.014. Epub 2017 May 8. PMID: 28495607.
28. Li CL, Tsuang YH, Tsai TH. Neuroprotective Effect of Schisandra Chinensis on Methyl-4Phenyl-1,2,3,6-Tetrahydropyridine-Induced

Parkinsonian Syndrome in C57BL/6 Mice. Nutrients. 2019;11(7):1671. Published 2019 Jul 21. doi:10.3390/nu11071671

29. A Mahfoz. Impacts of Immune System in an Experimental Social Behavior Model. Journal of Advanced Pharmacy Research, 2020, 4 (3), 72-82

30. Mahfoz, A. M. and N. Shahzad. Neuroinflammation impact in epileptogenesis and new treatment strategy. Behav Pharmacol., 2019, 30(8): 661-675.

31. Ali F. Abdel-Wahab, Mohamed A. Afify, Amal M. Mahfoz, Naiyer Shahzad, Ghazi A. Bamagous, Saeed S. Al Ghamdi. Vitamin D enhances antiepileptic and cognitive effects of lamotrigine in pentylenetrazole-kindled rats. Brain research, 2017, 1673: 78-85.

32. Amal M. Mahfoz, Ali F. Abdel-Wahab, Mohamed A. Afify, Naiyer Shahzad, Ibrahim A. A. Ibrahim, Naser A. ElSawy, Ghazi A. Bamagous, Saeed S. Al Ghamdi. Neuroprotective effects of vitamin D alone or in combination with lamotrigine against lithium-pilocarpine model of status epilepticus in rats

Naunyn-Schmiedeberg's Archives of Pharmacology (2017) 390:977-985. DOI 10.1007/s00210-017-1400-5. 